Draft version November 10, 2017

Typeset using $\mathrm{IAT}_{\mathrm{E}} \mathrm{X}$ modern style in AASTeX61

\title{
IMPLICATIONS FOR PLANETARY SYSTEM FORMATION FROM INTERSTELLAR OBJECT 1I/2017 U1 ('OUMUAMUA)
}

\author{
David E. Trilling, ${ }^{1}$ Tyler Robinson,${ }^{1}$ Alissa Roegge, ${ }^{1}$ \\ Colin Orion Chandler, ${ }^{1}$ Nathan Smith, ${ }^{1}$ Mark Loeffler,${ }^{1}$ Chad Trujillo, ${ }^{1}$ \\ Samuel Navarro-Meza, ${ }^{1,2}$ and Lori M. Glaspie ${ }^{1}$
}

${ }^{1}$ Department of Physics and Astronomy

Northern Arizona University

PO Box 6010

Flagstaff, AZ 86011

${ }^{2}$ Instituto de Astronomía

Universidad Nacional Autónoma de México

Ensendada B.C, 22860

México

(Received November 3, 2017; Revised November 8, 2017; Accepted November 8, 2017)

Submitted to Astrophysical Journal Letters

\begin{abstract}
The recently discovered minor body 1I/2017 U1 ('Oumuamua) is the first known object in our Solar System that is not bound by the Sun's gravity. Its hyperbolic orbit (eccentricity greater than unity) strongly suggests that it originated outside our Solar System; its red color is consistent with substantial space weathering experienced over a long interstellar journey. We carry out an simple calculation of the probability of detecting such an object. We find that the observed detection rate of 1I-like objects can be satisfied if the average mass of ejected material from nearby stars during the process of planetary formation is $\sim 20$ Earth masses, similar to the expected value for our Solar System. The current detection rate of such interstellar interlopers is estimated to be 0.2 /year, and the expected number of detections over the past few years is almost exactly one. When the Large Synoptic Survey Telescope begins its wide, fast, deep all-sky survey the detection rate will increase to 1 /year. Those expected detections will provide further constraints on nearby planetary system formation through a better estimate of the number and properties of interstellar objects.
\end{abstract}

Corresponding author: David E. Trilling

david.trilling@nau.edu 
Keywords: comets: individual (1I/2017 U1 ('Oumuamua)) - minor planets, asteroids: individual (1I/2017 U1 ('Oumuamua)) - protoplanetary disks - planetary systems - solar neighborhood - local interstellar matter 


\section{INTRODUCTION}

On October 18, 2017 the minor body C/2017-UT (PANSTARRS) that would become known as A/2017 U1 and later 1I/2017 U1 ('Oumuamua) - hereafter, 1I was discovered by the the Panoramic Survey Telescope And Rapid Response System (Pan-STARRS) survey (Chambers et al. 2016). In a matter of days it became clear that this object was on an unbound (hyperbolic) trajectory, with eccentricity $e \approx 1.2$ (Williams 2017). In addition to its high eccentricity, 1I's orbit is inclined to the Solar System plane at an angle $i \approx 123^{\circ}$, so it is very unlikely to have encountered any massive objects within our Solar System (other than the Sun). With no gravitational perturbations to explain its anomalously high eccentricity, the most likely explanation is that this object originated outside our Solar System and happened to pass close to the Earth during its journey through interstellar space. This implies that 1I formed in another planetary system and was ejected, presumably through dynamical interactions in its natal planetary system. Consequently, we refer to $1 \mathrm{I}$ and similar bodies as "ejectoids."

The theoretical existence of ejectoids has been long proposed (e.g., Sekanina 1976), with various authors using non-detections to place upper limits on the population (McGlynn \& Chapman 1989; Sen \& Rama 1993; Engelhardt et al. 2017). Jewitt (2003) and Francis (2005) suggested that PanSTARRS could detect an interstellar object, if the number density was great enough. In the two weeks since the discovery of $1 \mathrm{I}$ and its identification as an interstellar object, we have learned about its optical spectrum (Masiero 2017; Ye et al. 2017), its lightcurve (Knight et al. 2017), its orbit (de la Fuente Marcos \& de la Fuente Marcos 2017), and potential origin scenarios (Mamajek 2017; Gaidos et al. 2017; Laughlin \& Batygin 2017).

Here we use a simple calculation to estimate the probability of detecting such an object and explore the implications for the prevalence and properties of planetary systems that are implied by the existence and properties of $1 \mathrm{I}$.

\section{EJECTOID ENCOUNTERS AND EJECTED MASS CONSTRAINTS}

Gravitational microlensing results have shown that, on average, every star in the Milky Way is accompanied by at least one bound planet (Cassan et al. 2012), which implies that planet formation is a near-universal process. We assume here that the formation of a typical planetary system results in a mass $m$ of ejectoids with a typical size of $r_{\mathrm{e}}$ and mass density $\rho_{\mathrm{e}}$. The number of ejectoids per star is therefore

$$
\frac{m}{\frac{4}{3} \pi r_{\mathrm{e}}^{3} \rho_{\mathrm{e}}}
$$

We can write the number density of stars (in, e.g., stars per cubic parsec) $n_{\mathrm{s}}$ in terms of a characteristic stellar spacing, $R$, as

$$
n_{\mathrm{s}}=\frac{1}{\frac{4}{3} \pi R^{3}} \text {. }
$$


This enables us to express the number density of ejectoids (number/volume) as

$$
n_{\mathrm{e}}=\left(\frac{m}{\frac{4}{3} \pi r_{\mathrm{e}}^{3} \rho_{\mathrm{e}}}\right)\left(\frac{1}{\frac{4}{3} \pi R^{3}}\right) .
$$

We next wish to determine the number of ejectoids that we expect to have encountered. We assume that telescopic searches for 1I-like objects have swept out a cylindrical volume of interstellar space given by

$$
V_{\mathrm{obs}}=\pi r_{\mathrm{obs}}^{2} \Delta v_{\odot} \Delta t
$$

where $r_{\text {obs }}$ is the geocentric distance out to which we are sensitive to 1I-like objects, $\Delta v_{\odot}$ is the Sun's velocity through the solar neighborhood (and the presumed cloud of ejectoids), and $\Delta t$ is the time interval over which observational surveys have been capable of discovering 1I-like objects. Using this volume and the number density of ejectoids, we find that the number of detections of 1I-like objects is

$$
N=n_{\mathrm{e}} V_{\mathrm{obs}}=\left(\frac{m}{\frac{4}{3} \pi r_{\mathrm{e}}^{3} \rho_{\mathrm{e}}}\right)\left(\frac{1}{\frac{4}{3} \pi R^{3}}\right)\left(\pi r_{\mathrm{obs}}^{2} \Delta v_{\odot} \Delta t\right)
$$

which simplifies to

$$
N=\frac{9}{16 \pi} \frac{m r_{\mathrm{obs}}^{2} \Delta v_{\odot} \Delta t}{\rho_{\mathrm{e}} r_{\mathrm{e}}^{3} R^{3}} .
$$

We can now use 1I to constrain the characteristic mass in ejectoids for a forming planetary system. With $N=1$, we can re-arrange Equation 6 to write that

$$
m=\frac{16 \pi}{9} \frac{\rho_{\mathrm{e}} r_{\mathrm{e}}^{3} R^{3}}{r_{\mathrm{obs}}^{2} \Delta v_{\odot} \Delta t} .
$$

The absolute magnitude of $1 \mathrm{I}$ is given in the Minor Planet Center catalog as 22.1 (as of 2 November 2017), which corresponds to a radius $r_{e}$ of around 100 meters, assuming a moderate-to-dark albedo.

The range of densities for cometary and asteroidal material that may be relevant is around 500 to $3000 \mathrm{~kg} / \mathrm{m}^{3}$ (Richardson et al. 2007; Davidsson \& Gutiérrez 2006; Davidsson et al. 2007; Carry 2012). While 1I's highly eccentric orbit would be typically associated with a comet, and theoretical predictions suggest that most ejectoids should be more comet-like (Raymond et al. 2011), there are no signs of activity from 1I (Knight et al. 2017; Ye et al. 2017). Thus, here, we assume that 1I may be more asteroidal than cometary and adopt a density of $2000 \mathrm{~kg} / \mathrm{m}^{3}$.

There are 357 stars within 10 parsecs of the Sun $^{1}$. This gives an average distance between adjacent stars $R$ of 1.4 pc. The average discovery distance of Near Earth Objects in the Minor Planet Center $r_{\text {obs }}$ is 0.3 au.

${ }^{1}$ www.recons.org 
The velocity of the Sun relative to nearby stars is around $20 \mathrm{~km} / \mathrm{sec}$ (Schönrich et al. 2010). 1I has been found ${ }^{2}$ to have an interstellar speed (velocity at infinity) of $26 \mathrm{~km} / \mathrm{sec}$, while Mamajek (2017) reports that an object entering the Solar System with median velocity of the local stellar population would have a speed of around $22.5 \mathrm{~km} / \mathrm{sec}$. The fact that the Sun's relative velocity is comparable to the velocity of 1 I confirms our assumption that the population of interstellar ejectoids has zero mean velocity (due to the fact that both the source planetary systems and ejection trajectories are assumed to be isotropic). Based on these three estimates we set $\Delta v_{\odot}$ to be $25 \mathrm{~km} / \mathrm{sec}$.

In recent years improvements in detector size and field of view at the Catalina Sky Survey and Pan-STARRS (the two major NEO surveys) have enhanced the ability to detect 1I-like objects, so we estimate $\Delta t$ to be 5 years.

Our characteristic values, when inserted in Equation 7, yield a typical mass in ejected 1I-like objects of $10^{26} \mathrm{~kg}$, or $20 M_{\oplus}$. This is in remarkably good agreement with values derived for mass loss during the formation of our Solar System. For example, Weidenschilling (1977) and Bottke et al. (2005) derive $1-5 M_{\oplus}$ of material lost from the asteroid belt. Kuiper (1951), Kenyon \& Luu (1999), and Morbidelli (2005) find $12-30 M_{\oplus}$ lost from the Kuiper Belt. Together, these imply a total mass lost from our Solar System of close to $20 M_{\oplus}$.

Identifying the characteristic size and density of ejectoids as being the most uncertain terms in our analysis, and inserting the parameter values adopted above, we can write

$$
m=20 M_{\oplus}\left(\frac{\rho_{e}}{2000 \mathrm{~kg} \mathrm{~m}^{-3}}\right)\left(\frac{r_{e}}{100 \mathrm{~m}}\right)^{3} .
$$

This relationship is shown in Figure 1. The plausible range of ejection masses is roughly $1-100 M_{\oplus}$. We note that in principle the radius of $1 \mathrm{I}$, which we take to be characteristic of ejectoids, will be determined through our forthcoming thermal infrared observations of 1I with the Spitzer Space Telescope (observations scheduled for late November, 2017). There is no obvious way to constrain the density of $1 \mathrm{I}$.

Alternatively, if we adopt the $\sim 20 M_{\oplus}$ in ejectoids lost during the formation of the Solar System as a characteristic number, we can use Equation 6 to derive the number of $1 \mathrm{I}$-like objects expected over five years. We find that $\mathrm{N}$ is very close to unity - exactly matching the observations. The detection rate $(N / \Delta t)$ is therefore 0.2 1I-like objects per year, or one 1I-like object every five years. The number density of ejectoids is, from Equation 3, around $0.1 / \mathrm{pc}^{3}$.

\section{CAVEATS AND UNCERTAINTIES}

We do not claim that this is the only mechanism for producing 1I-like objects or delivering such objects to the detectable space near the Earth, as the above calculation

\footnotetext{
2 https://projectpluto.com/temp/2017u1.htm
} 
admittedly contains a number of assumptions. However, this does give a plausible explanation for 1I that in turn has several interesting implications that are discussed below.

The radius and density of 1I are unknown, though the values above are unlikely to be in error by more than a factor of two. Similarly, the geometry arguments (average stellar distance, solar velocity, observational distance) are likely within a factor of two, while the time interval is approximately correct. We ignore gravitational focusing here. The largest overall uncertainty is simply the unknown statistical likelihood of detecting this ejectoid and our extrapolation from a single object. 1I may be part of a constant stream of interstellar objects moving through our Solar System (as implied here), or a very unlikely occurrence, in which case the arguments made here are less applicable.

In addition, the true population of ejected extrasolar material must follow some size distribution, and will not consist of only $100 \mathrm{~m}$ 1I-like objects. Small objects are presumably more numerous in any planet formation scenario, but larger objects will be preferentially detected by our surveys. We must simply take $1 \mathrm{I}$ to be representative.

In the above calculation we have assumed that a steady state population of 1Ilike objects is ejected from all planetary systems. However, we might instead assume that the majority of ejectoids are produced during the earliest phases of planetary system formation, in a single pulse of material. The nearest star formation regions are some 100 parsec away, with typical ages of 1-10 Myr (Andrews et al. 2009; Currie \& Sicilia-Aguilar 2011; Esplin \& Luhman 2017). If we take the escape velocity from those systems to be on the order of $10 \mathrm{~km} / \mathrm{sec}$ then material from one of these nearby star formation regions would reach the Earth in a few million years, and we would therefore be moving through a cloud of ejected 1I-like objects. However, the rest of the assumptions still apply, and the expected value does not change significantly. A more complicated model (perhaps not warranted, given this single detection) could account for the total number of stars contained in the Milky Way, integrated over its history, as even stars that no longer exist could have contributed ejectoids to an interstellar stream of material.

Finally, we note that the above calculation implies a typical ejected mass of $20 M_{\oplus}$, but that need not imply that every stellar or planetary system ejects mass, or that amount of material. For example, while planet-planet scattering among gas giant planets is likely to produce a pulse of ejected planetesimals (Raymond et al. 2011; Marzari 2014), and while systems with gas giants on stable orbits can eject planetesimals on longer timescales (Raymond et al. 2011; Barclay et al. 2017), systems without gas giants rarely eject planetesimals because in that case escape velocity can not readily be achieved by planetesimals. Thus, if the fraction of nearby stars with gas giants is (for example) $50 \%$ then the average mass ejected by those systems must be a factor of $\sim 2$ greater than our nominal value in order to produce the necessary interstellar density of ejectoids. 


\section{IMPLICATIONS}

The 520-950 nm reflectance spectrum of 1I, albeit noisy, indicates no absorption features and a red spectral slope (Masiero 2017; Ye et al. 2017), making compositional characterization difficult. However, we note that a red spectral slope over this range is not unexpected and is characteristic of many primitive objects in our Solar System, (Cruikshank et al. 1998; Jewitt \& Luu 1999; Bus \& Binzel 2002; Sheppard 2010; Carry et al. 2016). Whether this slope is something intrinsic to the bulk properties of 1I or a consequence of its surface being altered via energetic processing is unclear. Given the presence of cosmic rays in the interstellar medium and other forms of ionizing radiation that would have been present in 1I's natal stellar environment, 1I's red spectral slope is entirely consistent with formation elsewhere and a long journey to our Solar System. This implies that the surface of 1I may have different properties than its bulk material.

1I's trajectory makes it very unlikely that it experienced a gravitational encounter with any of the proposed as-yet unknown planets in the outermost part of our Solar System (Trujillo \& Sheppard 2014; Brown \& Batygin 2016; Volk \& Malhotra 2017). Another possibility is that 1I was a member of our Solar System's Oort Cloud and was perturbed inbound onto an unbound orbit by a passing star. We do not comment on these scenarios; in this work we have assumed that $1 \mathrm{I}$ is an interstellar interloper that originated in a different plantary system.

The Large Synoptic Survey Telescope (LSST; Ivezić et al. 2008) will commence its ten year all-sky survey in 2022. One of the driving science cases for LSST is the detection of moving objects. Most moving objects detected by LSST will be "unremarkable" asteroids in the main belt, but this very large and deep survey $\left(20,000 \mathrm{deg}^{2}\right.$ surveyed to $r$ magnitude 24.5 repeatedly over ten years) naturally has the possibility to discover unusual objects in all areas of astrophysics. Several authors have studied the detectability of interstellar interlopers in LSST data (Moro-Martín et al. 2009; Cook et al. 2016; Engelhardt et al. 2017).

LSST will help constrain nearby planetary system formation by measuring the number of 1I-like objects. LSST will be sensitive to fainter and therefore smaller and/or more distant objects, and is therefore likely to have a greater detection rate than the current rate. The possibility of LSST detecting interstellar comets increases order(s) of magnitude when considering cometary outbursting (Cook et al. 2016), which makes objects brighter, although $1 \mathrm{I}$ has shown no signs of activity so far in its observational record.

As described above, with our current detection sensitivities the detection rate for 1I-like objects is $0.2 /$ year. The LSST detection limit will be around three magnitudes deeper than Pan-STARRS' typical limiting magnitude of $\mathrm{V} \sim 21.5$; this translates to a factor of three smaller in size. The only measured size distribution in this size range in our Solar System is the Near Earth Object population; a factor of three in size corresponds roughly to a factor of five in number of objects (Trilling et al. 2017). Thus, 
the expected detection rate of interstellar objects for LSST is around $1 /$ year. The LSST discovery rate of ejectoids will help us constrain the frequency and properties of planetary system formation in our nearby galaxy.

We thank an anonymous referee for a very prompt and thoughtful review that has improved this paper. This research has made use of data and/or services provided by the International Astronomical Union's Minor Planet Center. We have used information from the RECONS program, retrieved from www.recons.org. The discussions that led to this paper began at an NAU Astrocookies gathering.

\section{REFERENCES}

Andrews, S. M., Wilner, D. J., Hughes, A. M., Qi, C., \& Dullemond, C. P. 2009, ApJ, 700, 1502

Barclay, T., Quintana, E. V., Raymond, S. N., \& Penny, M. T. ApJ, 841, 86

Bottke, W. F., Durda, D. D., Nesvorný, D., et al. 2005, Icarus, 175, 111

Brown, M. E., \& Batygin, K. 2016, ApJL, 824, L23

Bus, S. J., \& Binzel, R. P. 2002, Icarus, 158, 146

Carry, B. 2012, Planet. Space Sci., 73, 98

Carry, B., Solano, E., Eggl, S., \& DeMeo, F. E. 2016, Icarus, 268, 340

Cassan, A., Kubas, D., Beaulieu, J. P., et al. 2012, Nature, 481, 167

Chambers, K. C., Magnier, E. A., Metcalfe, N., et al. 2016, arXiv.org, arXiv:1612.05560

Cook, N. V., Ragozzine, D., Granvik, M., \& Stephens, D. C. 2016, ApJ, 825, 51

Cruikshank, D. P., Roush, T. L.,

Bartholomew, M. J., et al. 1998, Icarus, 135,389

Currie, T., \& Sicilia-Aguilar, A. 2011, ApJ, 732, 24

Davidsson, B. J. R., \& Gutíerrez, P. J. 2006, Icarus, 180, 224

Davidsson, B. J. R., Gutíerrez, P. J., \& Rickman, H. 2007, Icarus, 187, 306

de la Fuente Marcos, C. \& de la Fuente Marcos, R. 2017, ArXiv e-prints, arXiv: 1711.00445

Engelhardt, T., Jedicke, R., Vereš, P., et al. 2017, AJ, 153, 133
Esplin, T. L., \& Luhman, K. L. 2017, AJ, 154, 134

Francis, P. J. 2005, ApJ, 635, 1348

Gaidos, E., Williams, J. P., \& Kraus, A. 2017, ArXiv e-prints, arXiv: 1711.01300

Ivezić, Ž., Tyson, J. A., Abel, B., et al. 2008, arXiv.org, 0805.2366v4

Jewitt, D. 2003, Earth Moon and Planets, 92,465

Jewitt, D., \& Luu, J. 1998, AJ, 115, 1667

Kenyon, S. J., \& Luu, J. X. 1999, AJ, 118, 1101

Knight, M. M., Protopapa, S., Kelly, M. S. P., et al. 2017, ArXiv e-prints, arXiv:1711.01402

Kuiper, G. P. 1951, in Proceedings of the National Academy of Sciences of the United States of America, Yerkes Observatory, University of Chicago, 114

Laughlin, G. \& Batygin, K. 2017, ArXiv e-prints, arXiv:1711.02260

Mamajek, E. 2017, ArXiv e-prints, arXiv:1710.11364

Marzari, F. 2014, MNRAS, 444, 1419

Masiero, J. 2017, ArXiv e-prints, arXiv:1710.09977

McGlynn, T. A., \& Chapman, R. D. 1989, ApJL, 346, L105

Morbidelli, A. 2005, ArXiv e-prints, arXiv:0512256

Moro-Martín, A., Turner, E. L., \& Loeb, A. 2009, ApJ, 704, 733

Raymond, S. N., Armitage, P. J., Moro-Martín, A., et al. 2011, A\&A, 530,62 
Richardson, J. E., Melosh, H. J., Lisse, C. M., \& Carcich, B. 2007, Icarus, 190, 357 Schönrich, R., Binney, J., \& Dehnen, W. 2010, MNRAS, 403, 1829

Sekanina, Z. 1976, Icarus, 27, 123

Sen, A. K., \& Rama, N. C. 1993, A\&A, 275,298

Sheppard, S. S. 2010, AJ, 139, 1394

Trilling, D. E., Valdes, F., Allen, L., et al. 2017, AJ, 154, 170

Trujillo, C. A., \& Sheppard, S. S. 2014, Nature, 507, 471
Volk, K., \& Malhotra, R. 2017, AJ, 154, 62

Weidenschilling, S. J. 1977, Astrophysics and Space Science, 51, 153

Williams, G. V. 2017, MPEC 2017-U183: A/2017 U1, https://www.minorplanetcenter.net/mpec/K17/K17UI3 Ye, Q.-Z., Zhang, Q., Kelley, M. S. P., \& Brown, P. G. 2017, ArXiv e-prints, arXiv:1711.02320 


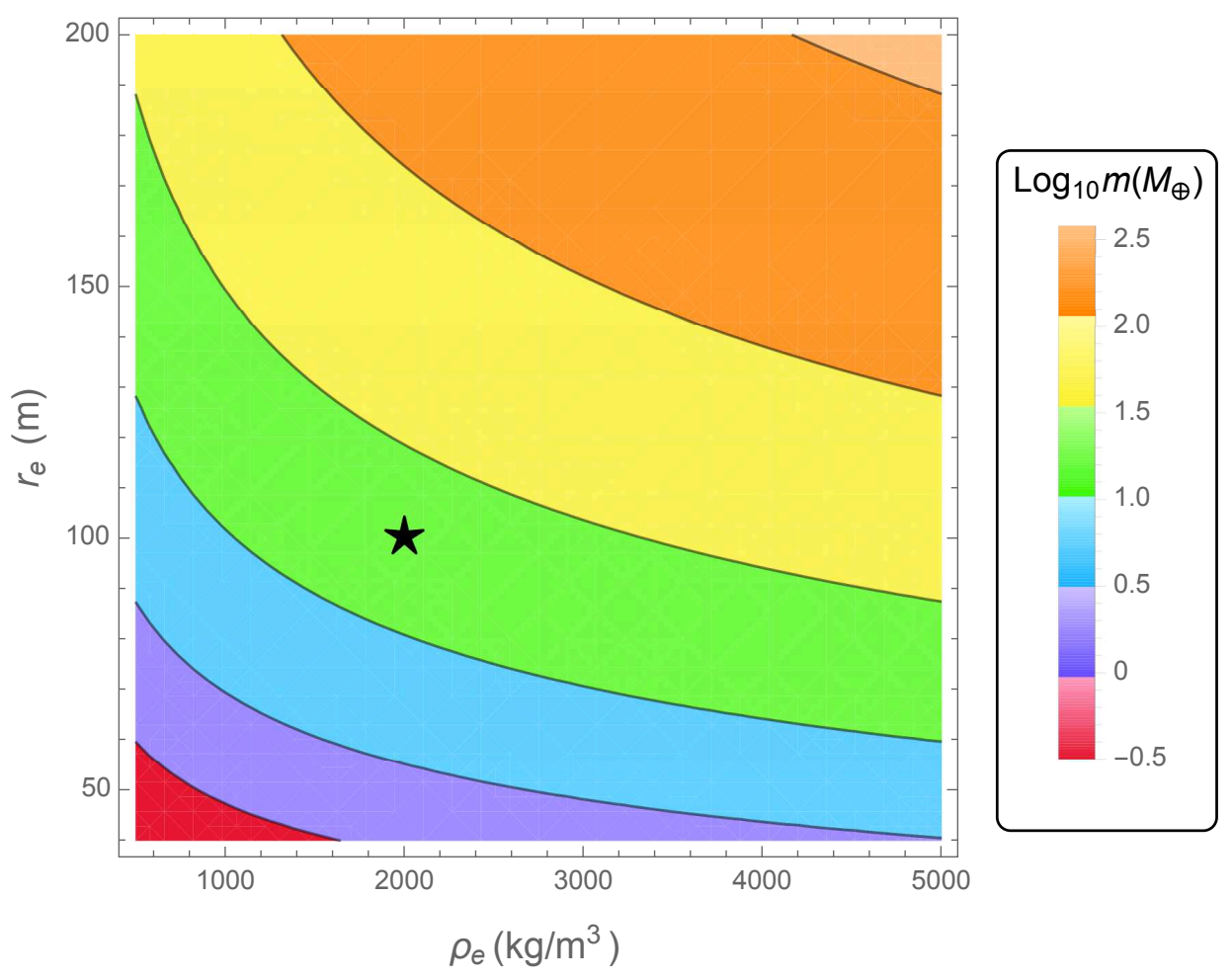

Figure 1. Characteristic ejection mass (colors and contours, in Earth masses) required to produce the observed rate of 1I-like objects as a function of the primary unknown parameters in our analysis: 1I's density $\left(\rho_{\mathrm{e}}\right)$ and radius $\left(r_{\mathrm{e}}\right)$. For nominal values of $2000 \mathrm{~kg} / \mathrm{m}^{3}$ and $100 \mathrm{~m}$, respectively, the required average ejection mass per star in the solar neighborhood is $20 M_{\oplus}$ (black star), remarkably close to various calculations of the mass lost from our Solar System during the era of planet formation. Our upcoming observations of 1I with the Spitzer Space Telescope in late November, 2017, should place a constraint on 1I's radius. 